\title{
Efeito do alumínio sobre compostos nitrogenados em Urochloa spp.
}

\author{
Lucas Anjos Souza ${ }^{1}$ \\ Liliane Santos Camargos ${ }^{2}$ \\ Leandro Ferreira Aguiar ${ }^{1 *}$ \\ ${ }^{1}$ Laboratório de Fisiologia Vegetal \\ Universidade Federal do Mato Grosso do Sul, Três Lagoas - MS, Brasil \\ ${ }^{2}$ Laboratório de Fisiologia do Metabolismo Vegetal, Departamento de Biologia e Zootecnia \\ Universidade Estadual Paulista Júlio de Mesquita Filho, Faculdade de Engenharia de Ilha Solteira \\ Passeio Monção, 226, CEP 15385-000, Ilha Solteira - SP, Brasil \\ * Autor para correspondência \\ 1faguiar@pq.cnpq.br
}

\section{Resumo}

A superfície terrestre apresenta, em sua composição, aproximadamente 7\% de alumínio (Al), na forma de óxidos de alumínio ou aluminossilicatos, entretanto, sua disponibilidade é extremamente dependente do $\mathrm{pH}$ do solo. O cerrado é um bioma que apresenta solos com $\mathrm{pH}$ baixo e possui grande quantidade de $\mathrm{Al}^{+3}$ disponível; dessa forma, a produção vegetal pode se tornar limitada. Os vegetais possuem mecanismo para tolerar quantidades excessivas de $\mathrm{Al}^{+3}$ no solo; um dos mecanismos utilizados para esse propósito é a exsudação de ácidos orgânicos, que são capazes de formar complexos com o $\mathrm{Al}^{+3}$, e, assim, ele se torna indisponível para as plantas. Uma vez disponível, o $\mathrm{Al}^{+3}$ pode afetar o metabolismo de nitrogênio $(\mathrm{N})$, levando a perdas na produção. Portanto, este estudo teve por objetivo avaliar o efeito do $\mathrm{Al}^{+3}$ sobre compostos relacionados ao metabolismo de $\mathrm{N}$ em três espécies do gênero Urochloa (U. brizantha, U. decumbens e $U$. humiducola). O delineamento experimental foi inteiramente casualizado em esquema fatorial $2 \times 3$ (com e sem $\mathrm{Al}^{+3}$ e 3 espécies de Urochloa); a dose de $\mathrm{Al}^{+3}$ aplicada foi de $200 \mu$ mol.L $L^{-1}$ em solução com pH de 4,5. Dentre as espécies avaliadas não foram observadas alterações na concentração de compostos relacionados ao metabolismo de $\mathrm{N}$ em resposta ao $\mathrm{Al}^{+3}$, o que pode sugerir a existência de mecanismos de adaptação que permitem a essas plantas manter o metabolismo de $\mathrm{N}$ inalterado mesmo diante de situação de estresse.

Palavras-chave: Cerrado brasileiro; Indicadores de estresse; Nitrogênio; Tolerância; Toxicidade

\section{Abstract}

Aluminum effect on nitrogenous compounds in Urochloa spp. Earth's surface has, in its composition, around $7 \%$ of aluminum (Al), in the form of aluminum oxides or aluminosilicates, however, its availability is greatly dependent on soil $\mathrm{pH}$. Cerrado is a biome presenting low $\mathrm{pH}$ soils and it has high amounts of $\mathrm{Al}^{+3}$ available; thus, plant production may become limited. Plants have mechanism to tolerate excessive amounts of $\mathrm{Al}^{+3}$ in soil; one of the mechanisms used for this purpose is the exudation of organic acids, which are able to form complexes with $\mathrm{Al}^{+3}$, and, thus, it becomes unavailable to plants. Once available, $\mathrm{Al}^{+3}$ can affect nitrogen 
(N) metabolism, leading to production losses. Therefore, this study aimed to evaluate the effect of $\mathrm{Al}^{+3}$ on compounds related to $\mathrm{N}$ metabolism in 3 species from the genus Urochloa (U. brizantha, U. decumbens, and $U$. humiducola). The experimental design was completely randomized in a $2 \times 3$ factorial scheme (with and without $\mathrm{Al}^{+3}$ and 3 species of Urochloa); the dose of $\mathrm{Al}^{+3}$ applied was $200 \mu \mathrm{mol}$. $\mathrm{L}^{-1}$ in a solution with $\mathrm{pH}$ 4.5. Among the species evaluated, no changes were observed in compound content concentrations related to $\mathrm{N}$ metabolism as a response to $\mathrm{Al}^{+3}$, something which may suggest the existence of adaptation mechanisms that allow these plants to keep $\mathrm{N}$ metabolism unaltered even in face of a stressful situation.

Key words: Brazilian cerrado; Nitrogen; Stress indicators; Tolerance; Toxicity

\section{Introdução}

O cerrado, um dos maiores biomas brasileiros, possui solos ácidos (BUOL, 2009), e essa condição favorece a disponibilização de quantidades excessivas de $\mathrm{Al}^{+3}$, que é fator limitante à produção de culturas nesses solos (DELHAIZE; RYAN, 1995). O Al ${ }^{+3}$ inibe o crescimento radicular, dessa forma prejudica a exploração do solo e a absorção de água e nutrientes (MEDA; FURLANI, 2005).

A tolerância ao $\mathrm{Al}^{+3}$ pode ocorrer por meio de dois mecanismos que são amplamente conhecidos: exclusão do $\mathrm{Al}^{+3}$ absorvido ou armazenamento de modo quelado (KOCHIAN et al., 2004) e ambos os mecanismos permitem às plantas tolerarem $\mathrm{o} \mathrm{Al}^{+3} \mathrm{do}$ solo. A exposição ao $\mathrm{Al}^{+3}$ pode induzir a liberação de ácidos orgânicos que protegem as raízes contra $\mathrm{o} \mathrm{Al}^{+3}$ quelando esse elemento na rizosfera e então, culminando na formação de complexos não tóxicos (RYAN et al., 2001). Espécies que toleram altas concentrações de $\mathrm{Al}^{+3}$ geralmente usam mais eficientemente o mecanismo de complexação na rizosfera em detrimento da acumulação.

$\mathrm{O} \mathrm{N}$ é um elemento essencial ao crescimento vegetal; e é absorvido do solo como nitrato, amônia, ureia ou aminoácidos (WILLIAMS; MILLER, 2001), sendo o nitrato a forma preferencialmente absorvida. Bactérias quimiossintetizantes dos gêneros Nitrosomonas e Nitrobacter oxidam amônia e nitrito, respectivamente, e usam os elétrons provenientes dessa oxidação em seu metabolismo. $\mathrm{O} \mathrm{Al}^{+3}$ pode causar efeitos drásticos na assimilação de $\mathrm{N}$ em plantas (PAL'OVE-BALANG; MISTRIK, 2011); o que leva ao declínio da concentração de nitrato na presença de $\mathrm{Al}^{+3}$ que, segundo Keltjens e Vanulden (1987), é devido à extrema inibição da absorção do nitrato pelas raízes.
Plantas do gênero Urochloa são originárias do continente africano e foram introduzidas no Brasil. Muitas espécies de Urochloa que foram introduzidas no Brasil podem sair de controle e invadir áreas cultivadas, competindo com espécies nativas. As espécies mais agressivas nesse quesito são: $B$. brizantha, $B$. decumbens, $B$. humidicola e $B$. mutica. Sendo que as duas últimas ocorrem frequentemente em lagos e são capazes de eliminar totalmente a flora nativa.

Já se sabe que essas espécies são tolerantes à toxidez do $\mathrm{Al}^{+3}$, mas não há estudos acerca do funcionamento do metabolismo de $\mathrm{N}$ em situações de estresse por $\mathrm{Al}^{+3}$; e considerando a importância econômica desse gênero para a produção animal, o presente estudo teve como objetivo avaliar se há diferenças quanto à influência do $\mathrm{Al}^{+3}$ no metabolismo do $\mathrm{N}$ entre as espécies de Urochloa spp. estudadas.

\section{Material e Métodos}

Sementes de três espécies de Urochloa ( $U$. brizantha, $U$. decumbens e $U$. humidicola) foram semeadas em tubetes contendo vermiculita, recebendo água destilada diariamente até a germinação. Após a germinação, as plântulas receberam, em dias alternados, solução nutritiva completa de Hoagland e Arnon (1950) a $10 \%$ de força iônica; 30 dias após crescerem nos tubetes, as plântulas foram transferidas para vasos com capacidade de $1 \mathrm{~L}$ contendo vermiculita; a partir desse momento as plantas passaram a receber $100 \mathrm{~mL}$ de solução nutritiva completa de Hoagland e Arnon (1950) a $50 \%$ de força iônica, em intervalos de dois dias até atingirem o tamanho ideal para aplicação do tratamento. A manutenção hídrica foi realizada diariamente. 
$\mathrm{O}$ delineamento experimental foi inteiramente casualizado em esquema fatorial $2 \times 3\left(\mathrm{sem} \mathrm{Al}^{+3}\right.$ e com $\mathrm{Al}^{+3}$ e três espécies de Urochloa) com três repetições biológicas individuais para cada tratamento. Os tratamentos foram identificados como: $\mathrm{T}_{1}(U$. brizantha sem $\left.\mathrm{Al}^{+3}\right), \mathrm{T}_{2}\left(U\right.$. brizantha com $\left.\mathrm{Al}^{+3}\right), \mathrm{T}_{3}(U$. decumbens sem $\left.\mathrm{Al}^{+3}\right), \mathrm{T}_{4}\left(U\right.$. decumbens com $\left.\mathrm{Al}^{+3}\right), \mathrm{T}_{5}$ (U. humidicola $\mathrm{sem} \mathrm{Al}^{+3}$ ) e $\mathrm{T}_{6}\left(U\right.$. humidicola $\mathrm{com} \mathrm{Al}^{+3}$ ). A solução nutritiva acrescida de $200 \mu \mathrm{mol} . \mathrm{L}^{-1}$ de $\mathrm{Al}^{+3}$ foi aplicada nos tratamentos $\mathrm{T}_{2}, \mathrm{~T}_{4}$ e $\mathrm{T}_{6}$. Quatro dias após a aplicação dos tratamentos, folhas e raízes foram coletadas e imediatamente congeladas em $\mathrm{N}_{2}$ líquido para caracterização bioquímica dos compostos de interesse.

A extração de proteínas solúveis totais foi realizada utilizando-se solução de hidróxido de sódio $(\mathrm{NaOH})$ a 0,1 M na proporção de 1:10 (m/v). Após a homogeneização, a mistura foi centrifugada e o sobrenadante foi coletado e armazenado em freezer para posterior quantificação proteica.

Compostos nitrogenados foram extraídos segundo o método descrito por Bieleski e Turner (1966) usando solução de metanol, clorofórmio e água (MCW) na proporção de 12:5:3 (v/v/v), respectivamente. A extração foi realizada homogeneizando os tecidos em solução MCW na proporção 1:10 (m/v), o material já homogeneizado foi centrifugado durante 15 minutos a $3000 \mathrm{rpm}$. A cada $1 \mathrm{~mL}$ de sobrenadante recuperado foi adicionada $0,25 \mathrm{~mL}$ de clorofórmio e $0,67 \mathrm{~mL}$ de água destilada, e então essa mistura foi agitada vigorosamente e deixada em repouso a $4^{\circ} \mathrm{C}$ durante $24 \mathrm{~h}$; então a fase aquosa/metanólica foi coletada e utilizada para quantificação de aminoácidos solúveis totais, nitrato, ureídeos totais e ácido alantoico.

A quantificação de proteínas solúveis totais foi realizada de acordo com o método proposto por Bradford (1976), usando albumina sérica bovina (BSA) como padrão para construção da curva analítica. A quantificação de aminoácidos solúveis totais foi realizada de acordo com o método proposto por Yemm e Cocking (1955), usando glicina como padrão para construção da curva analítica. A quantificação de nitrato foi realizada pelo método proposto por Cataldo et al. (1975), usando nitrato de potássio como padrão para construção da curva analítica. A quantificação de ureídeos totais e ácido alantoico foi realizada pelo método proposto por Vogels e Van der Drift (1970), usando alantoína como padrão para construção da curva analítica. A quantificação de alantoína foi realizada calculando-se a diferença entre a concentração de ureídeos totais e a concentração de ácido alantoico.

A análise estatística foi realizada por meio do software SISVAR ${ }^{\circledR}$, e as médias foram comparadas pelo teste de Tukey a $5 \%$ de probabilidade

\section{Resultados e Discussão}

Alguns autores têm avaliado a resposta de grande variedade de plantas expostas ao $\mathrm{Al}^{+3}$ (CRUZ et al., 2011; MACEDO et al., 2011), entretanto, poucos têm usado abordagens com caracterização bioquímica sobre o estresse ocasionado por esse elemento. Avaliações das concentrações de diversos compostos mostram que alguns metabólitos não foram afetados pela presença de $\mathrm{Al}^{+3}$ nas espécies de Urochloa estudadas. As três espécies apresentaram concentrações similares de nitrato em folhas, enquanto que em raízes $U$. brizantha aprensetou concentração menor que $U$. decumbens e $U$. humidicola (Figura 1 A e 1 B).

As observações acerca da concentração de nitrato em raízes e folhas nos permitem sugerir que nessas espécies a enzima redutase do nitrato pode não ser diretamente influenciada pela presença de $\mathrm{Al}^{+3}$ ou ainda, pode sugerir mecanismo relacionado à assimilação de $\mathrm{N}$ que pode conferir tolerância a essa via; e apesar da similaridade das respostas entre as espécies ainda são necessários mais estudos para compreendermos o possível mecanismo atuante e se o mesmo é igual ou similar entre as espécies do grupo. Esses resultados podem estar relacionados à resposta diferenciada da enzima redutase do nitrato, que geralmente apresenta sensibilidade ao $\mathrm{Al}^{+3}$, como já demonstrado por alguns autores (JUSTINO et al., 2006; CRUZ et al., 2011).

A concentração de aminoácidos e proteínas solúveis totais são parâmetros bioquímicos confiáveis que indicam a condição fisiológica das plantas em diversas situações de estresse que essas podem estar sujeitas (AZMAT; KHAN, 2011). Cruz et al. (2011) observaram que a presença do $\mathrm{Al}^{+3}$ ocasiona a diminuição da concentração 
FIGURA 1: Concentração de nitrato ( $\mu$ mol.g-1 de massa fresca) em folhas (A) e raízes (B) de três espécies de Urochloa na ausência e presença de $\mathrm{Al}^{+3}$. Letras minúsculas comparam as médias entre as espécies na ausência e presença de $\mathrm{Al}^{+3}$; letras maiúsculas comparam a resposta de cada espécie individualmente na ausência e presença de $\mathrm{Al}^{+3}$. Letras iguais não diferem pelo teste de Tukey a 5\% de probabilidade.

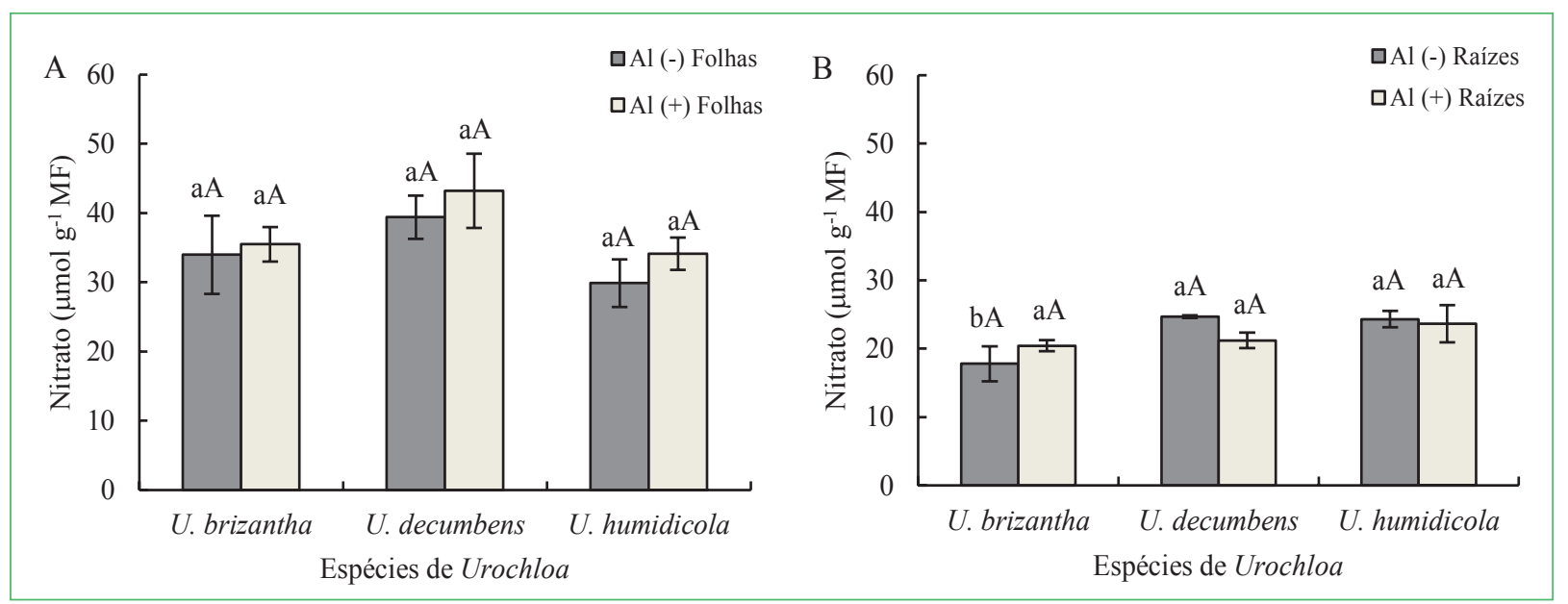

de aminoácidos e proteínas solúveis totais em plantas de sorgo, mostrando que a presença desse elemento pode, de fato, limitar o desenvolvimento vegetal. Entretanto, os resultados do presente trabalho demonstram que nas espécies de Urochloa estudadas não houve alterações na concentração de aminoácidos e de proteínas em plantas expostas ao $\mathrm{Al}^{+3}$ (Figura $2 \mathrm{~A}$ e $2 \mathrm{~B}$ ) e (Figura $3 \mathrm{~A}$ e 3 $\mathrm{B})$, sendo que as diferenças observadas na concentração proteica foram apenas entre as espécies, sugerindo que a disponibilidade destes compostos pode variar dependendo da espécie, e que espécies tolerantes podem não apresentar decréscimo nas concentrações desses compostos mantendo assim a homeostase metabólica. É interessante observar que plantas do gênero Urochloa são usadas como pasto para criação de diversos animais, assim a sua capacidade de crescer em solos ácidos reflete diretamente na possibilidade de expansão pecuária de regiões ricas em solos ácidos.

Foi possível observar diferenças na concentração de ureídeos totais apenas entre as espécies, mas não em função do $\mathrm{Al}^{+3}$; sendo que raízes foram os órgãos que apresentaram maiores quantidades desses compostos (Figura 4 A e 4 B).

FIGURA 2: Concentração de aminoácidos solúveis totais ( $\mu$ mol.g-1 de massa fresca) em folhas (A) e raízes (B) de três espécies de Urochloa na ausência e presença de $\mathrm{Al}^{+3}$. Letras minúsculas comparam as médias entre as espécies na ausência e presença de $\mathrm{Al}^{+3}$; letras maiúsculas comparam a resposta de cada espécie individualmente na ausência e presença de $\mathrm{Al}^{+3}$. Letras iguais não diferem pelo teste de Tukey a 5\% de probabilidade.

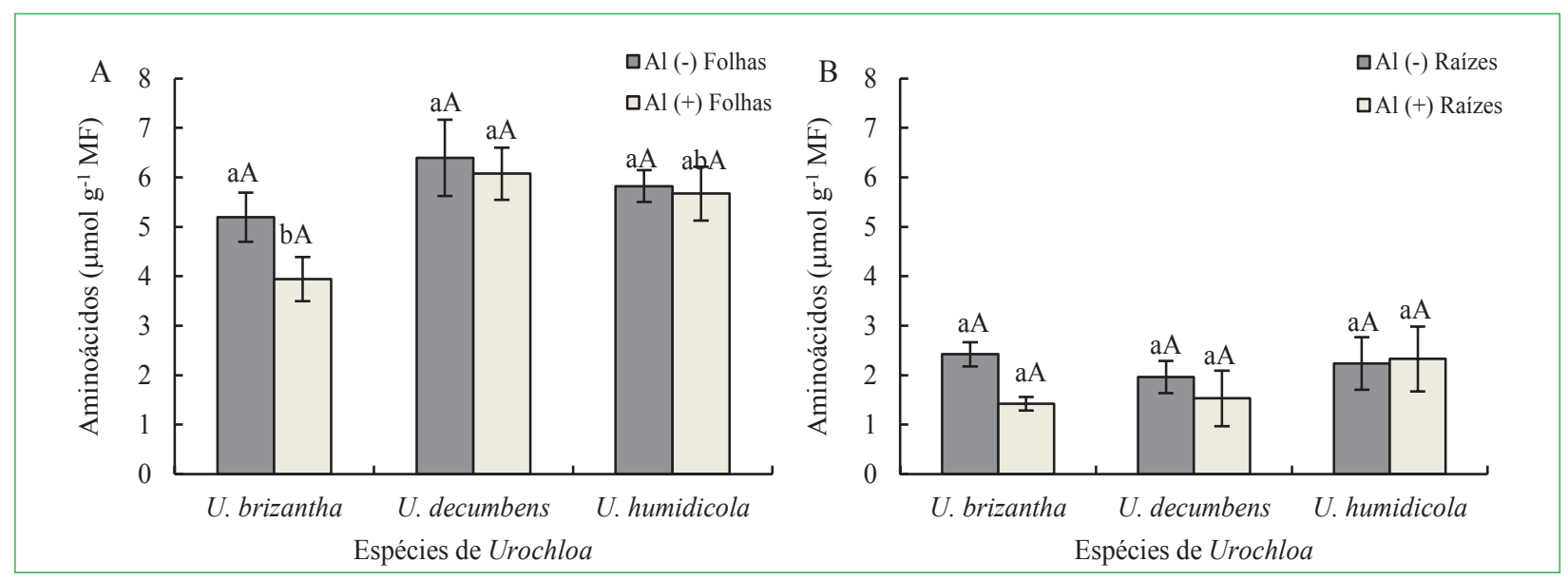


FIGURA 3: Concentração de proteínas solúveis totais ( $\mathrm{mg} \mathrm{g}^{-1}$ de massa fresca) em folhas (A) e raízes (B) de três espécies de Urochloa na ausência e presença de $\mathrm{Al}^{+3}$. Letras minúsculas comparam as médias entre as espécies na ausência e presença de $\mathrm{Al}^{+3}$; letras maiúsculas comparam a resposta de cada espécie individualmente na ausência e presença de $\mathrm{Al}^{+3}$. Letras iguais não diferem pelo teste de Tukey a $5 \%$ de probabilidade.

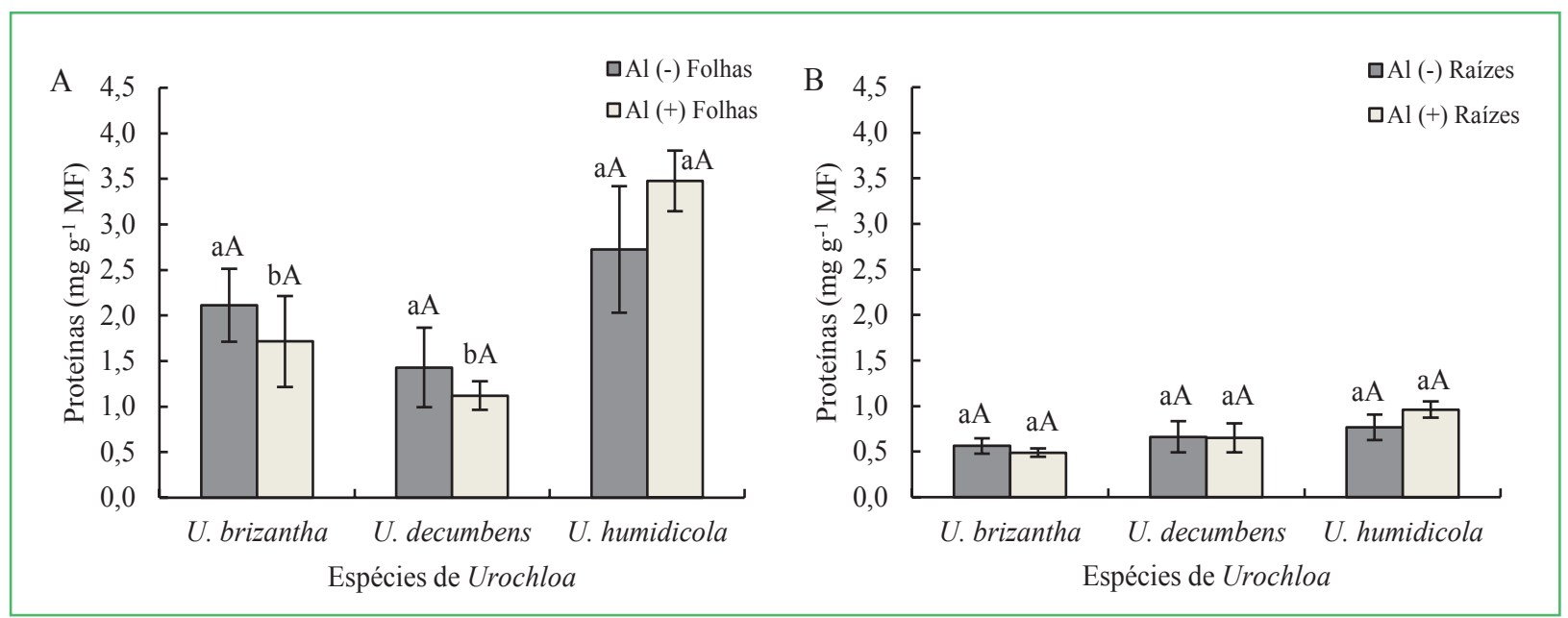

FIGURA 4: Concentração de ureídeos totais ( $\mu$ mol. $\mathrm{g}^{-1}$ de massa fresca) em folhas (A) e raízes (B) de três espécies de Urochloa na ausência e presença de $\mathrm{Al}^{+3}$. Letras minúsculas comparam as médias entre as espécies na ausência e presença de $\mathrm{Al}^{+3}$; letras maiúsculas comparam a resposta de cada espécie individualmente na ausência e presença de $\mathrm{Al}^{+3}$. Letras iguais não diferem pelo teste de Tukey a $5 \%$ de probabilidade.

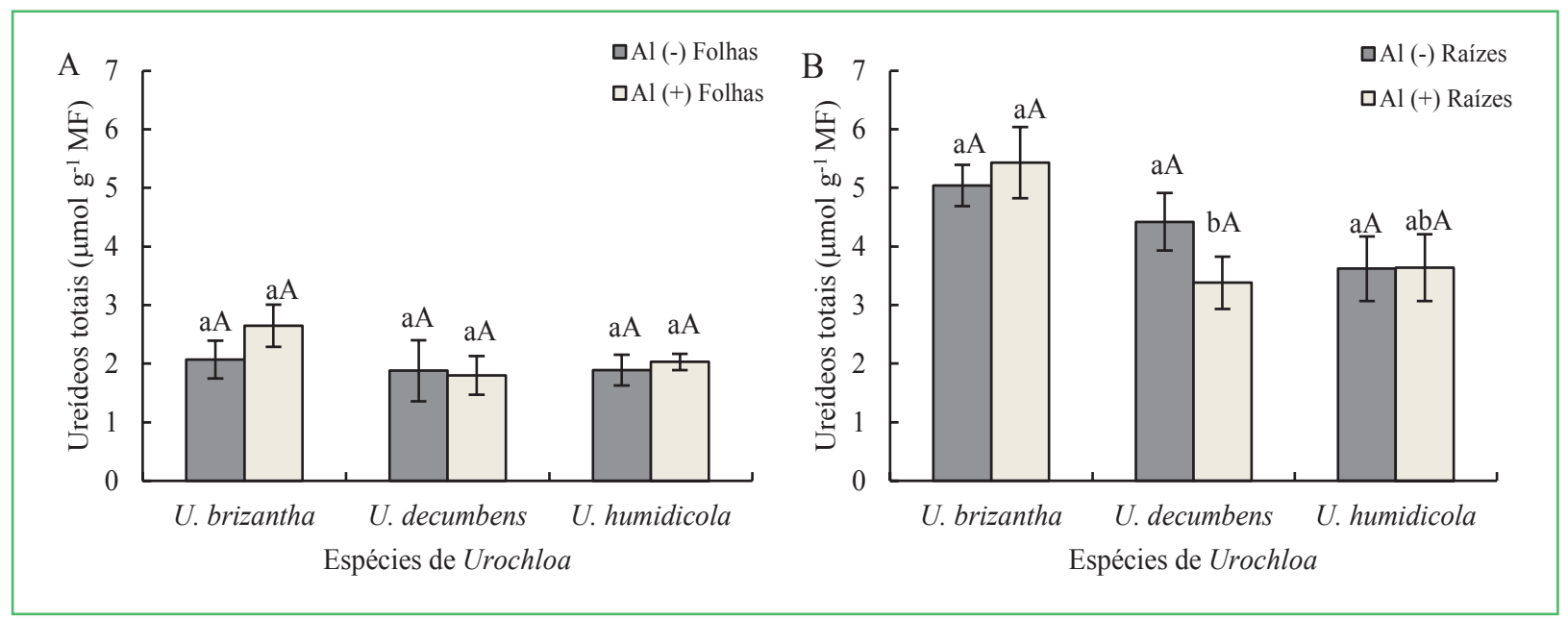

Essa observação pode ser fator indicativo sobre a existência de alterações no metabolismo dessa classe de composto na presença de $\mathrm{Al}^{+3}$ além de mostrar que a resposta é diferente entre as espécies; entretanto há necessidade de estudos mais aprofundados para estabelecermos o grau de envolvimento dessa classe de compostos na adaptação ao estresse ocasionado pelo $\mathrm{Al}^{+3}$.
Os ureídeos investigados no presente estudo, ácido alantoico e alantoína, são compostos nitrogenados que, geralmente, estão associados à fixação biológica de $\mathrm{N}$ em plantas leguminosas (AMARANTE; SODEK, 2006). Entretanto, esses compostos vêm sendo detectados em plantas não leguminosas e sua produção pode estar relacionada à degradação de purinas, como relatado 
por Todd et al. (2006). Adicionalmente, o acúmulo desses compostos pode estar diretamente relacionada a aquisição de tolerância em situações estressantes, como tem sido reportado por alguns autores (SINCLAIR et al., 2003; PURCELL et al., 2004; KING; PURCELL, 2005). Portanto, o fato de não observarmos nenhuma alteração significativa nessa classe de compostos (Figura $5 \mathrm{~A} \mathrm{e} 5 \mathrm{~B}$ ) e (Figura $6 \mathrm{~A}$ e $6 \mathrm{~B}$ ) em resposta ao $\mathrm{Al}^{+3}$ pode indicar estabilidade metabólica e sugerir mecanismo de adaptação que esteja relacionado a esses compostos.
Diante do exposto, pode-se concluir que a estabilidade metabólica da classe de compostos avaliados frente ao estresse imposto pela presença de $\mathrm{Al}^{+3}$ permite sugerir que as espécies estudadas apresentaram boa capacidade de crescimento em solos ácidos, em grande parte pela capacidade de utilizar normalmente o N disponível no solo mesmo sobre o forte estresse ocasionado pela presença do $\mathrm{Al}^{+3}$.

FIGURA 5: Concentração de ácido alantoico ( $\mu$ mol.g $\mathrm{g}^{-1}$ de massa fresca) em folhas (A) e raízes (B) de três espécies de Urochloa na ausência e presença de $\mathrm{Al}^{+3}$. Letras minúsculas comparam as médias entre as espécies na ausência e presença de $\mathrm{Al}^{+3}$; letras maiúsculas comparam a resposta de cada espécie individualmente na ausência e presença de $\mathrm{Al}^{+3}$. Letras iguais não diferem pelo teste de Tukey a $5 \%$ de probabilidade.

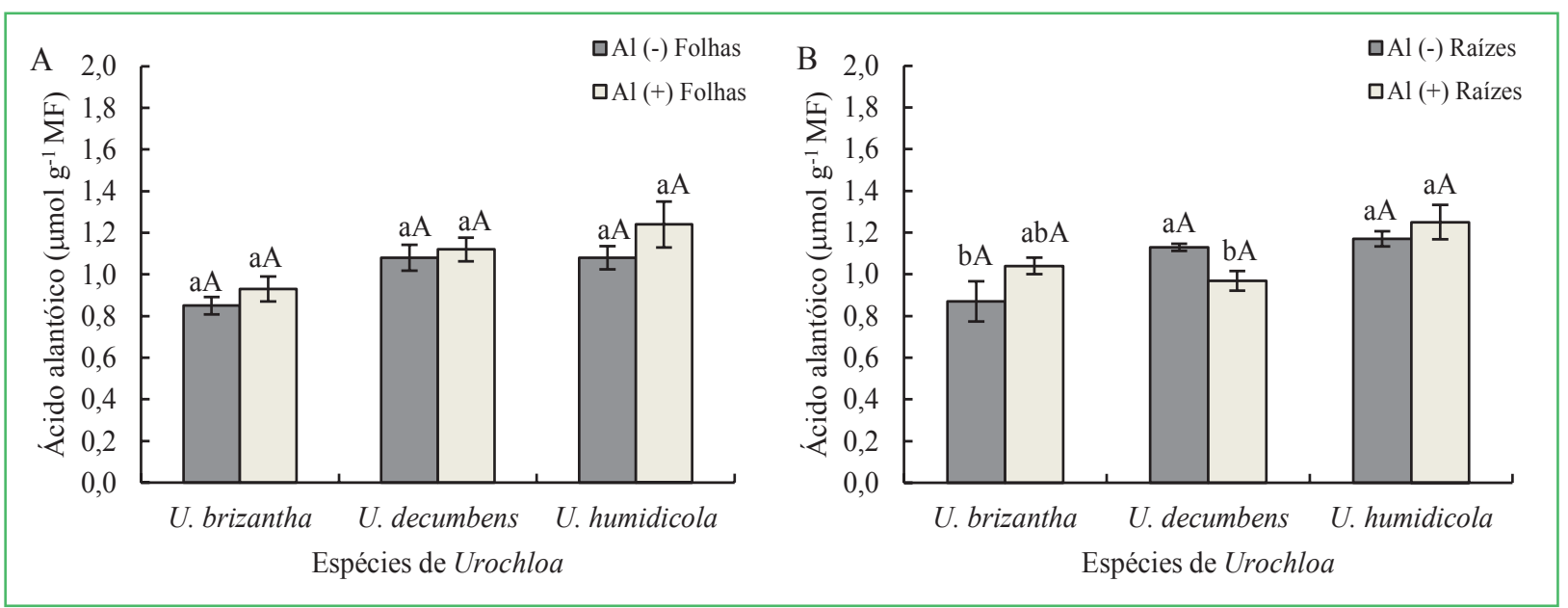

FIGURA 6: Concentração de alantoína ( $\mu$ mol.g-1 de massa fresca) em folhas (A) e raízes (B) de três espécies de Urochloa na ausência e presença de $\mathrm{Al}^{+3}$. Letras minúsculas comparam as médias entre as espécies na ausência e presença de $\mathrm{Al}^{+3}$; letras maiúsculas comparam a resposta de cada espécie individualmente na ausência e presença de $\mathrm{Al}^{+3}$. Letras iguais não diferem pelo teste de Tukey a $5 \%$ de probabilidade.

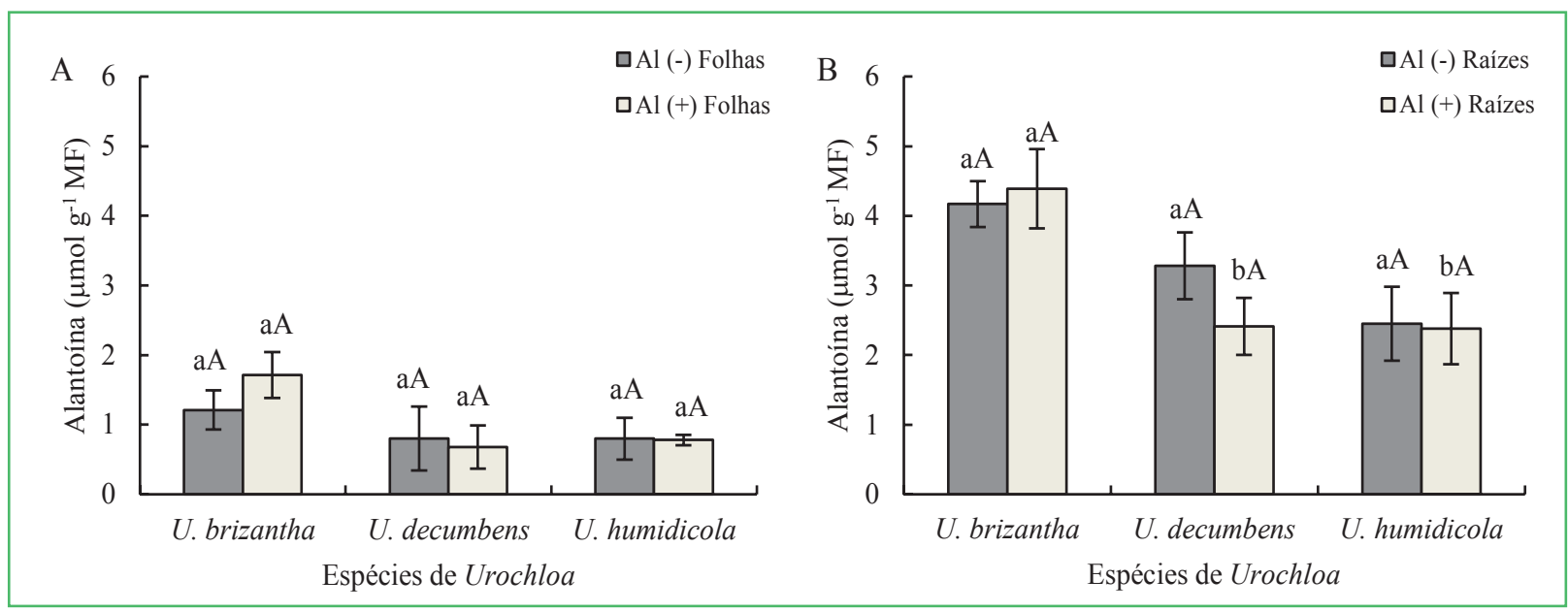




\section{Agradecimentos}

Os autores gostariam de deixar seus agradecimentos ao $\mathrm{CNPq}$ e ao Fundect pelo apoio financeiro para realização da presente pesquisa.

\section{Referências}

AMARANTE, L.; SODEK, L. Waterlogging effect on xylem sap glutamine of nodulated soybean. Biologia Plantarum, Dordrecht, v. 50, n. 3, p. 405-410, 2006.

AZMAT, R.; KHAN, N. Nitrogen metabolism as a bioindicator of $\mathrm{Cu}$ stress in Vigna radiata. Pakistan Journal of Botany, Karachi, v. 43, n. 1, p. 515-520, 2011.

BIELESKI, R. L.; TURNER, N. A. Separation and estimation of amino acids in crude plant extracts by thin-layer electrophoresis and chromatography. Analytical Biochemistry, San Diego, v. 17 n. 2, p. 278-293, 1966.

BRADFORD, M. M. Rapid and sensitive method for quantitation of microgram quantities of protein utilizing principle of proteindye binding. Analytical Biochemistry, San Diego, v. 72, n. 1-2, p. 248-254, 1976.

BUOL, S. W. Soils and agriculture in central-west and north Brazil. Scientia Agricola, Piracicaba, v. 66, n. 5, p. 697-707, 2009.

CATALDO, D. A.; HAROON, M.; SCHRADER, L. E.; YOUNGS, V. L. Rapid colorimetric determination of nitrate in plant-tissue by nitration of salicylic-acid. Communications in Soil Science and Plant Analysis, Philadelphia, v. 6, n. 1, p. 71-80, 1975.

CRUZ, F. J. R.; LOBATO, A. K. S.; COSTA, R. C. L.; LOPES, M. J. S.; NEVES, H. K. B.; NETO, C. F. O.; SILVA, M. H. L.; FILHO, B. G. S.; JUNIOR, J. A. L.; OKUMURA, R. S. Aluminum negative impact on nitrate reductase activity, nitrogen compounds and morphological parameters in sorghum plants. Australian Journal of Crop Science, Lismore, v. 5, n. 6, p. 641-645, 2011.

DELHAIZE, E.; RYAN, P. R. Aluminum toxicity and tolerance in plants. Plant Physiology, Rockville, v. 107, n. 2, p. 315-321, 1995. JUSTINO, G. C.; CAMBRAIA, J.; OLIVA, M. A.; OLIVEIRA, J. A. Absorção e redução de nitrato em duas cultivares de arroz na presença de alumínio. Pesquisa Agropecuária Brasileira, Brasília, v. 41, n. 8, p. 1285-1290, 2006.

HOAGLAND, D. R.; ARNON, D. I. The water-culture method for growing plants without soil. Berkeley: California Agricultural Experimental Station Bulletin, 1950. 32 p.
KELTJENS, W. G.; VANULDEN, P. S. R. Effects of Al on nitrogen $\left(\mathrm{NH}_{4}^{+}\right.$and $\left.\mathrm{NO}_{3}^{-}\right)$uptake, nitrate reductase activity and proton release in 2 sorghun cultivars differing in $\mathrm{Al}$ tolerance. Plant and Soil, Dordrecht, v. 104, n. 2, p. 227-234, 1987.

KING, C. A.; PURCELL, L. C. Inhibition of $\mathrm{N}_{2}$ fixation in soybean is associated with elevated ureides and amino acids. Plant Physiology, Rockville, v. 137, n. 4, p. 1389-1396, 2005.

KOCHIAN, L. V.; HOEKENGA, O. A.; PINEROS, M. A. How do crop plants tolerate acid soils? Mechanisms of aluminum tolerance and phosphorous efficiency. Annual Review of Plant Biology, Palo Alto, v. 55, n. 1, p. 459-493, 2004.

MACEDO, C. M. P.; LOPES, J. C.; AMARAL, J. A. T.; FONSECA, A. F. A.; AMARAL, J. F. T. Tolerance of arabica coffee cultivars for aluminum in nutritive solution. Brazilian Archives of Biology and Technology, Curitiba, v. 54, n. 5, p. 885-891, 2011.

MEDA, A. R.; FURLANI, P. R. Tolerance to aluminum toxicity by tropical leguminous plants used as cover crops. Brazilian Archives of Biology and Technology, Curitiba, v. 48, n. 2, p. 309-317, 2005. PAL'OVE-BALANG, P.; MISTRIK, I. Effect of aluminium on nitrogen assimilation in roots of Lotus japonicus. Plant Biosystems, Oxon, v. 145, n. 3, p. 527-531, 2011.

PURCELL, L. C.; SERRAJ, R.; SINCLAIR, T. R.; DE, A. Soybean $\mathrm{N}_{2}$ fixation estimates, ureide concentration, and yield responses to drought. Crop Science, Madison, v. 44, n. 2, p. 484-492, 2004.

RYAN, P. R.; DELHAIZE, E.; JONES, D. L. Function and mechanism of organic anion exudation from plant roots. Annual Review of Plant Physiology and Plant Molecular Biology, Palo Alto, v. 52, n. 1, p. 527-560, 2001.

SINCLAIR, T. R.; VADEZ, V.; CHENU, K. Ureide accumulation in response to Mn nutrition by eight soybean genotypes with $\mathrm{N}_{2}$ fixation tolerance to soil drying. Crop Science, Madison, v. 43, n. 2, p. 592-597, 2003.

TODD, C. D.; TIPTON, P. A.; BLEVINS, D. G.; PIEDRAS, P.; PINEDA, M.; POLACCO, J. C. Update on ureide degradation in legumes. Journal of Experimental Botany, Oxford, v. 57, n. 1, p. 5-12, 2006.

VOGELS, G. D.; VAN DER DRIFT, C. Differential analyses of glyoxylate derivatives. Analytical Biochemistry, San Diego, v. 33, n. 1, p. 143-157, 1970.

WILLIAMS, L. E.; MILLER, A. J. Transporters responsible for the uptake and partitioning of nitrogenous solutes. Annual Review of Plant Physiology and Plant Molecular Biology, Palo Alto, v. 52, n. 1, p. 659-692, 2001.

YEMM, E. W.; COCKING, E. C. The determination of amino acids with ninhydrin. Analyst, Cambridge, v. 80, n. 948, p. 209213, 1955. 